\title{
Rare Case of Adult Congenital Cystic Adenomatoid Malformation Diagnosed with CT Perfusion Imaging and Hystological Sample
}

\author{
Gianluigi Sergiacomi ${ }^{1}$, Costantino Del Giudice ${ }^{1}$, Amedeo Ferlosio², Mara Mataloni',

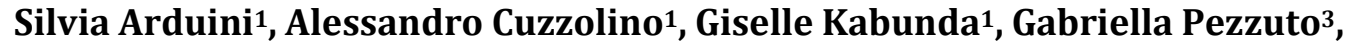 \\ Augusto Orlandi ${ }^{2}$, Giovanni Simonetti ${ }^{1}$ \\ ${ }^{1}$ Department of Diagnostic Imaging, Molecular Imaging, Interventional Radiology and Radiation Therapy, IRCCS \\ Policlinico Tor Vergata, Rome, Italy \\ ${ }^{2}$ Anatomic Pathology Institute, Department of Biomedicine and Prevention, IRCCS Policlinico Tor Vergata, \\ Rome, Italy \\ ${ }^{3}$ Department of Internal Medicine, IRCCS Policlinico Tor Vergata, Rome, Italy \\ Email: costantino.delgiudice@gmail.com
}

Received 18 March 2014; revised 18 April 2014; accepted 25 April 2014

Copyright @ 2014 by authors and Scientific Research Publishing Inc.

This work is licensed under the Creative Commons Attribution International License (CC BY). http://creativecommons.org/licenses/by/4.0/

(c) (i) Open Access

\begin{abstract}
We describe the case of a 44-year-old man with congenital cystic adenomatoid malformation. Both computed tomographic (CT) scan findings with perfusion protocol and histological biopsy led to diagnosis. CT scan showed multiple terminal bronchiolar-like uniform cysts smaller than $2 \mathrm{~cm}$ and CT perfusion allowed to make a differential diagnosis with an usual interstitial pneumonia. In order to make a definite diagnosis, an open lung biopsy confirmed the CT findings.
\end{abstract}

Keywords

Congenital Cystic Adenomatoid Malformation, Computed Tomography, Perfusion

\section{Introduction}

Congenital cystic adenomatoid malformation (CCAM) is a developmental anomaly, with a reported incidence of 1 in 25.000 - 35.000 pregnancies [1]. This malformation is characterized by a maldevelopment of terminal branches due to an abnormal embryogenesis during the first 6 - 7 weeks of pregnancy [2]. Ch'in and Tang de- 
scribed this disease for the first in 1949 [3] and classified into 3 histological types by Stocker in 1977.

Usually the first diagnosis is made by ultrasound examination in the neonatal period. Rare cases are diagnosed in adulthood, often as a result of the onset of complications, such as pneumonia or abscesses.

We describe a case of adult patient with Stocker's Type II CAM in who the diagnosis was performed with the support of computed tomography (CT) perfusion imaging and histological biopsy.

\section{Case Report}

A 44-year-old boy presented to our hospital with progressive dyspnea on exertion and dry cough without fever over 6 years. Chest exam revealed bibasilar inspiratory crackles at the both lung bases. Pulmonary function examination showed a mild to moderate decrease of mixed type ventilation function and diffusion function. No serological markers for autoimmune disease were detected. Optical fibro-bronchoscopy (FOB) and BAL were done in to rule out infection: total cell count was $690.000 \mathrm{cell} / \mathrm{ml}$, and cell differential counts were as follows: epithelial cells $15 \%$, alveolar macrophages $79 \%$, lymphocytes $4 \%$. A volumetric unenhanced breath-hold CT scan of the chest using a 64-slice multi-detector CT scanner (LightSpeed 64; General Electric Company, Fairfield, CT, USA) was performed with the following parameters: $100 \mathrm{kV} ; 120 \mathrm{mAs}$; detector configuration, $24 \times$ $1.2 \mathrm{~mm}$; rotation time, 0.3 second; section thickness, $3.0 \mathrm{~mm}$; reconstruction interval, $3 \mathrm{~mm}$; reconstruction kernel, B30 and B60. The study showed a cystic reversal of the parenchyma, with the presence of bronchiectasis smaller than $2 \mathrm{~cm}$. This framework was localized at the level of the lingula, the middle lobe and both lower lobes. Some portions of adjacent parenchyma presented a ground-glass aspect. The remaining portions of the lung parenchyma appeared unharmed (Figure 1). A Usual interstitial pneumonia (UIP) was suspected and the patient was started on corticosteroid therapy with ensuing progression of symptoms and functional and radiological deterioration. In order to put a differential diagnosis with an usual interstitial pneumonia and non-specific interstitial pneumonia a dynamic CT perfusion. Using a 64-slice multi-detector CT scanner a new unenhanced chest CT scan was performed, with the same parameters previously described, in order to evaluate the location of the parenchimal cystic subversion. After injection of $80 \mathrm{ml}$ of high-concentration (350 mg per milliliter of iodine) nonionic iodinated contrast medium (Iomeron 350; Bracco, Milan, Italy) through a 18-gauge cannula placed into a superficial vein of the antecubital fossa, free-breathing dynamic acquisition $(100 \mathrm{kV}$; detector configuration, $24 \times 1.2$; rotation time, 0.33 second; section thickness, $3.0 \mathrm{~mm}$; reconstruction increment, 3 $\mathrm{mm}$; total scanning time, 70 seconds; four-dimensional range, $136 \mathrm{~mm} / 1.75 \mathrm{sec}$ per scan; total scans, 40; reconstruction kernel, B30). The quality reference was set at $120 \mathrm{mAs}$ by using an automated tube current modulation (Auto mA; General Electric Company, Fairfield, CT, USA). The dynamic study showed an area of pulmonary parenchima with reduced anarchic blood volume (mean $6.6 \pm 9.7 \mathrm{ml} / 100 \mathrm{ml}$ ), time to peak (mean $5.8 \pm$ 1.8 seconds) and blood flow (mean $72.9 \pm 111.9 \mathrm{ml} / 100 \mathrm{ml} / \mathrm{min}$ ) distribution. The adjacent groud-glass paren-

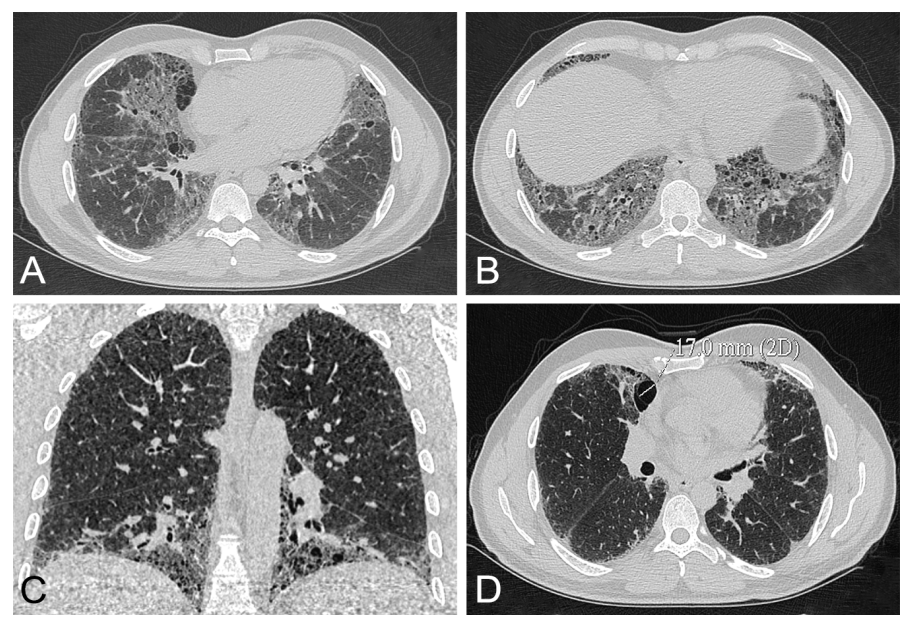

Figure 1. Multi-detector CT imaging in axial (A) (B) (D) and coronal (C) view showing the cystic reversal of the parenchyma, with the presence of bronchiectasis smaller than $2 \mathrm{~cm}$ extended to the lingual, the middle and both lower lobes. 
chimal area showed an increased homogenous blood volume $(75.4 \pm 9.2 \mathrm{ml} / 100 \mathrm{ml})$ time to peak (mean $11.6 \pm$ 0.7 seconds) and flow distribution (mean $394.5 \pm 70.9 \mathrm{ml} / 100 \mathrm{ml} / \mathrm{min}$ ) (Figure 2). A preliminary diagnosis of atypical congenital cystic adenomatoid malformation was performed.

A surgical biopsy was performed to confirm the diagnosis (Figure 3). The histological analysis of pulmonary lingula sample showed the absence of normal lung tissue replaced by an anarchic tissue constituted by cystic dilatation of bronchiolar structures lined by proliferation of cuboidal or ciliated columnar epithelial cells along the alveoli and respiratory bronchioles with focal exudates and necrotic debris filled in some dilated cysts and increased smooth muscle and stromal elastica change. The histological data associated to the radiological images allowed a final diagnosis of Type II cystic adenomatoid malformation. He remained asymptomatic till we contacted him for follow-up at two years.

\section{Discussion}

The CCAM is part of the group of structural abnormalities collectively referred to as bronchopulmonary foregut
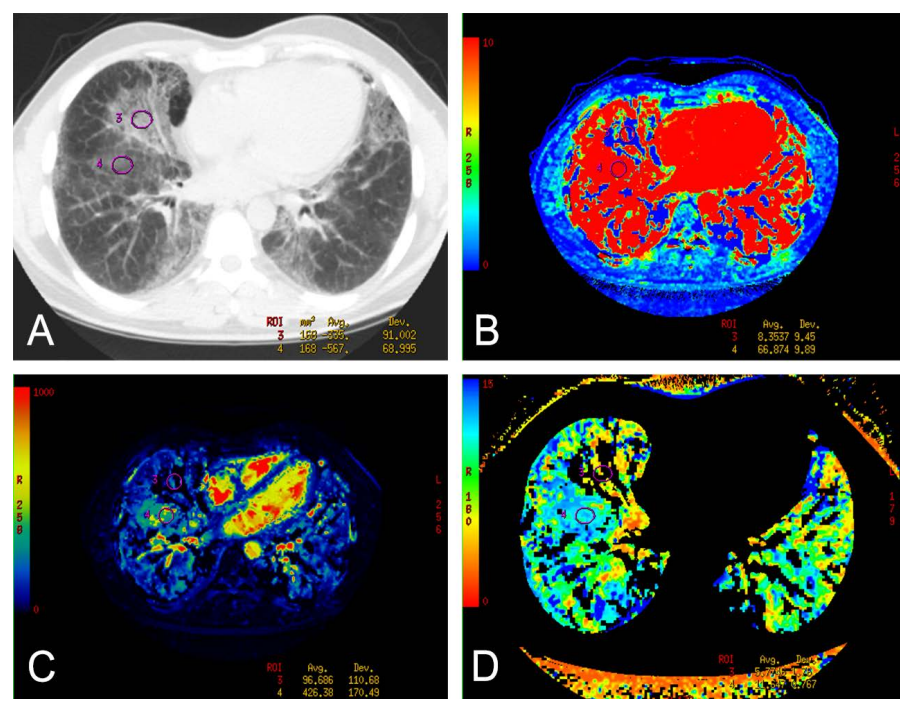

Figure 2. Volumetric (A) and dynamic perfusion CT imaging (B)-(D) showing the anarchic perfusion parameters of the involved parenchima. Volume (B) and flow (C) mapping show an anarchic reduced blood perfusion with a peripheral ground-glass parenchimal area characterized by an increased blood volume and flow. The mean transit time (D) mapping shows a reduced transit time in the cystic reversal area with a reduced transit time in the adjacent ground-glass parenchimal area.
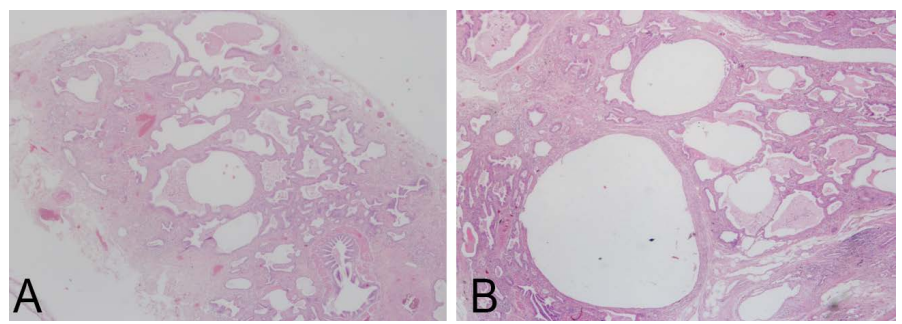

Figure 3. Histological representative pictures showing the absence of normal lung tissue replaced by cystic dilatation of bronchiolar structures (A) lined by proliferation of cuboidal or ciliated columnar epithelial cells along the alveoli and respiratory bronchioles with focal exudates and necrotic debris filled in some dilated cysts and increased smooth muscle and stromal elastica change (B) (Haematoxilin-Eosin stain, original magnification $200 \times$ and $400 \times$, respectively). 
malformations due to altered development of the respiratory system at 3 weeks. The classical histological change of CCAM is a replacement of normal pulmonary alveoli by cyst composed of adenomatoid hyperplastic bronchioles. In 1977, Stocker described a first classification of this disease subdividing in three groups. Type I CCAM is characterized by single or multiple cysts more than $3 \mathrm{~cm}$ in diameter lined by pseudostratified ciliated columnar epithelium, along with mucous cells. Type II lesion is consisted of multiple terminal bronchiolar-like uniform cysts smaller than $2 \mathrm{~cm}$ in diameter, lined by cuboidal to columnar epithelium. Type III CCAM usually involves an entire lobe of lung and has a spongy-like appearance, constructed by bulk gland-like structures. Usually CCAM is diagnosed in the first two years of life [4] manifesting as respiratory distress in neonates or recurrent infections in older children. In adult presentation of CCAM is very rare, with few cases documented in the literature [5]-[7]. Clinical manifestation may vary from a totally asymptomatic presentation to recurrent infectious events.

Usually under clinical suspect as in our case, further exams are performed, as pulmonary functional test and broncoscopy, that results aspecific. Diagnosis usually is performed by the combination of CT exam and histological findings. In our case, the chest CT scan findings were inconclusive due to the presence of cystic reversal with cyst smaller than $2 \mathrm{~cm}$ associated to a ground glass aspect. Moreover the malformation didn't involve a whole lobe but only segment of middle on lower lobe. In order to avoid a pulmonary biopsy to a young patient an attempt to perform a symptomatic therapy with corticosteroid was performed without success.

Recently perfusion CT has been introduced as an evolving functional imaging technique that is able to generate estimates of tissue perfusion and related parameters. At the beginning this technique was applied on oncologic imaging, where perfusion CT may assist in understanding the tumors characteristics and provide interesting information to asses an efficacious therapy [8] and stroke imaging to assess the magnitude and extent of ischemic damage [9]. In the last years several groups have demonstrated the utility of this technique to describe chronic lung disease, obtaining functional information about the pulmonary parenchima that couldn't be achieved with traditional diagnostic imaging [10]. In our case, a dynamic study was used to evaluated the different vascular pattern of this disease and obtain a differential diagnosis. Usually an increased peripheral vascularization with elevated blood volume, blood flow and reduced time to peak was observed in a UIP. The central area of the lesion on the contrary is characterized by a reduced vascularization. In our case an anarchic flow and blood volume was observed due to the abnormal cystic distribution. Our findings were successfully confirmed by a subsequent lung biopsy that showed the presence of anarchic tissue organized in cystic dilatation smaller than $2 \mathrm{~cm}$ and lined by ciliated columnar cells as in Stocker type II CCAM. Diagnosis is important not only to perform an adeguate treatment of possible secondary complications as hemorrhage and recurrent infection, but also because a strict follow-up is necessary for the possible development of malignancies due to the pseudostratified ciliated columnar epithelium and the mucous cells that lines the cysts which are considered to potentially mutant. In fact rhabdomyosarcomas, pulmonary blastomas, bronchioloalveolar carcinomas, and squamous cell carcinomas have all been described [11].

\section{Conclusion}

CCAM in adults may have atypical clinical and radiological manifestation. Dynamic CT findings could be useful to lead to the diagnosis before histological biopsy.

\section{References}

[1] Laberge, J.M., Flageole, H., Pugash, D., Khalife, S., Blair, G., Filiatrault, D., Russo, P., Lees, G. and Wilson, R.D. (2001) Outcome of the Prenatally Diagnosed Congenital Cystic Adenomatoid Lung Malformation: A Canadian Experience. Fetal Diagnosis and Therapy, 16, 178-186. http://dx.doi.org/10.1159/000053905

[2] Adzick, N.S., Harrison, M.R., Flake, A.W., Howell, L.J., Golbus, M.S. and Filly, R.A. (1993) Fetal Surgery for Cystic Adenomatoid Malformation of the Lung. Journal of Pediatric Surgery, 28, 806-812. http://dx.doi.org/10.1016/0022-3468(93)90332-F

[3] Ch’In, K.Y. and Tang, M.Y. (1949) Congenital Adenomatoid Malformation of One Lobe of a Lung with General Anasarca. Archives Pathology (Chic), 48, 221-229.

[4] Luck, S.R., Reynolds, M. and Raffensperger, J.G. (1986) Congenital Bronchopulmonary Malformations. Current Problems in Surgery, 23, 245-314. http://dx.doi.org/10.1016/0011-3840(86)90013-4

[5] Plit, M.L., Blott, J.A., Lakis, N., Murray, J. and Plit, M. (1997) Clinical, Radiographic and Lung Function Features of 
Diffuse Congenital Cystic Adenomatoid Malformation of the Lung in an Adult. The European Respiratory Journal, 10, 1680-1682. http://dx.doi.org/10.1183/09031936.97.10071680

[6] Han, Y.M., Lee, D.K., Lee, S.Y., Chung, K.H., Kim, M.H., Sohn, M.H., Kim, C.S. and Choi, K.C. (1994) Adult Presentation of Congenital Cystic Adenomatoid Malformation of the Lung: A Case Report. Journal of Korean Medical Science, 9, 86-91.

[7] Avitabile, A.M., Greco, M.A., Hulnick, D.H. and Feiner, H.D. (1984) Congenital Cystic Adenomatoid Malformation of the Lung in Adults. The American Journal of Surgical Pathology, 8, 193-202. http://dx.doi.org/10.1097/00000478-198403000-00005

[8] Ng, Q.S., Goh, V., Fichte, H., Klotz, E., Fernie, P., Saunders, M.I., Hoskin, P.J. and Padhani, A.R. (2006) Lung Cancer Perfusion at Multi-Detector Row CT: Reproducibility of Whole Tumor Quantitative Measurements. Radiology, 239, 547-553. http://dx.doi.org/10.1148/radiol.2392050568

[9] Thierfelder, K.M., Sommer, W.H., Baumann, A.B., Klotz, E., Meinel, F.G., Strobl, F.F., Nikolaou, K., Reiser, M.F. and von Baumgarten, L. (2013) Whole-Brain CT Perfusion: Reliability and Reproducibility of Volumetric Perfusion Deficit Assessment in Patients with Acute Ischemic Stroke. Neuroradiology, 55, 827-835. http://dx.doi.org/10.1007/s00234-013-1179-0

[10] Alford, S.K., van Beek, E.J., McLennan, G. and Hoffman, E.A. (2010) Heterogeneity of Pulmonary Perfusion as a Mechanistic Image-Based Phenotype in Emphysema Susceptible Smokers. Proceedings of the National Academy of Sciences of the United States of America, 107, 7485-7490. http://dx.doi.org/10.1073/pnas.0913880107

[11] Benouaich, V., Marcheix, B., Begueret, H., Brouchet, L., Velly, J.F. and Jougon, J. (2009) Malignancy of Congenital Cystic Adenomatoid Malformation of Lung in Aged. Asian Cardiovascular \& Thoracic Annals, 17, 634-636. http://dx.doi.org/10.1177/0218492309349810 